\title{
Silicon optomechanical crystal resonator at millikelvin temperatures
}

\author{
Seán M. Meenehan, ${ }^{1}$ Justin D. Cohen, ${ }^{1}$ Simon Gröblacher, ${ }^{1,2}$ Jeff T. Hill,,${ }^{1}$ Amir H. Safavi-Naeini, ${ }^{1}$ \\ Markus Aspelmeyer, ${ }^{2}$ and Oskar Painter ${ }^{1, *}$ \\ ${ }^{1}$ Institute for Quantum Information and Matter and Thomas J. Watson, Sr., Laboratory of Applied Physics, \\ California Institute of Technology, Pasadena, California 91125, USA \\ ${ }^{2}$ Vienna Center for Quantum Science and Technology (VCQ), Faculty of Physics, University of Vienna, A-1090 Wien, Austria
}

(Received 24 March 2014; published 17 July 2014)

\begin{abstract}
Optical measurements of a nanoscale silicon optomechanical crystal cavity with a mechanical resonance frequency of $3.6 \mathrm{GHz}$ are performed at subkelvin temperatures. We infer optical-absorption-induced heating and damping of the mechanical resonator from measurements of phonon occupancy and motional sideband asymmetry. At the lowest probe power and lowest fridge temperature $\left(T_{\mathrm{f}}=10 \mathrm{mK}\right)$, the localized mechanical resonance is found to couple at a rate of $\gamma_{\mathrm{i}} / 2 \pi=400 \mathrm{~Hz}\left(Q_{\mathrm{m}}=9 \times 10^{6}\right)$ to a thermal bath of temperature $T_{\mathrm{b}} \approx 270 \mathrm{mK}$. These measurements indicate that silicon optomechanical crystals cooled to millikelvin temperatures should be suitable for a variety of experiments involving coherent coupling between photons and phonons at the single quanta level.
\end{abstract}

DOI: 10.1103/PhysRevA.90.011803

PACS number(s): 42.50.Wk, 42.65.-k, 62.25.-g

The coupling of a mechanical object's motion to the electromagnetic field of a high finesse cavity forms the basis of various precision measurements [1], from large-scale gravitational wave detection [2] to microscale accelerometers [3]. Recent work utilizing both optical and microwave cavities coupled to mesoscopic mechanical resonators has shown the capability to prepare and detect such resonators close to their quantum ground state of motion using radiation pressure backaction [4-7]. Optomechanical crystals (OMCs), in which band gaps for both optical and mechanical waves can be introduced through patterning of a material, provide a means for strongly interacting nanomechanical resonators with near-infrared light [8]. Beyond the usual paradigm of cavity optomechanics involving isolated single mechanical elements [9,10], OMCs can be fashioned into planar circuits for photons and phonons, and arrays of optomechanical elements can be interconnected via optical and acoustic waveguides [11]. Such coupled OMC arrays have been proposed as a way to realize quantum optomechanical memories [12], nanomechanical circuits for continuous variable quantum information processing [13] and phononic quantum networks [14], and as a platform for engineering and studying quantum many-body physics of optomechanical metamaterials [15-17].

The realization of optomechanical systems in the quantum regime is predicated upon the ability to limit thermal noise in the mechanics while simultaneously introducing large coherent coupling between optical and mechanical degrees of freedom. In this regard, laser back-action cooling has recently been employed in simple OMC cavity systems [6,18] consisting of a one-dimensional (1D) nanobeam resonator surrounded by a two-dimensional (2D) phononic band gap. In this work, we optically measure the properties of such an OMC cavity system in a helium dilution refrigerator down to base temperatures of $T_{\mathrm{f}}=10 \mathrm{mK}$.

The device studied here is formed from the top silicon (Si) device layer of a silicon-on-insulator (SOI) wafer [19]. Figure 1(a) shows a scanning electron microscope (SEM)

*opainter@caltech.edu image of a suspended device after processing. This device consists of two nanobeam OMC cavities, optically coupled to a common central waveguide [Fig. 1(b)]. As described in Ref. [6], the nanobeam cavities are patterned in such a way as to support an optical resonance in the $1550 \mathrm{~nm}$ wavelength band and a "breathing" mechanical resonance at 3.6 GHz. The highly localized optical and acoustic resonances couple strongly via radiation pressure, with a theoretical vacuum coupling rate of $g_{0} / 2 \pi=870 \mathrm{kHz}$, corresponding physically to the optical resonance shift due to the mechanical resonator zero-point amplitude. Surrounding the waveguide and nanobeam structure on three sides is a 2D "cross" pattern [11] which has a full phononic band gap for all acoustic waves in the 3-4 GHz frequency range. The fourth side is left open, so as to allow close approach of an optical fiber. The SOI sample is mounted to the mixing chamber plate of a dilution refrigerator, and a set of stages are used to align an antireflection-coated tapered lensed fiber (beam waist $=2.5 \mu \mathrm{m}$; focal distance $=14 \mu \mathrm{m}$ ) to the coupling waveguide of a given device under test [see Fig. 1(a) and Ref. [19]]. In order to aid efficient optical coupling, the Si waveguide is tapered down to a tip of width $225 \mathrm{~nm}$, providing mode matching between fiber and waveguide [20,21].

Optical spectroscopy of a fiber-coupled device at a fridge temperature of $4 \mathrm{~K}$ is shown in Fig. 1(c). In this measurement, the frequency of a narrow linewidth external-cavity diode laser is swept across the fundamental optical resonance of one of the OMC cavities centered at $\lambda_{\mathrm{c}}=1545 \mathrm{~nm}$. The input laser light is reflected from a photonic crystal mirror at the end of the Si waveguide, and when resonant, evanescently couples in to the nanobeam cavities (the other nanobeam cavity coupled to this waveguide has a resonance several $\mathrm{THz}$ to the red). Some of the light entering the cavity decays through intrinsic loss channels at rate $\kappa_{\mathrm{i}}$, while the remainder couples back into the central waveguide at coupling rate $\kappa_{\mathrm{e}}$, and is collected in reflection by the lensed optical fiber. For this particular cavity, we observe a total optical energy decay rate of $\kappa / 2 \pi=529 \mathrm{MHz}$, an extrinsic coupling rate $\kappa_{\mathrm{e}} / 2 \pi=153 \mathrm{MHz}$, and an intrinsic decay rate $\kappa_{\mathrm{i}} / 2 \pi=376 \mathrm{MHz}$. From the normalized reflection signal level $(R)$, the fraction of optical power reflected by the 


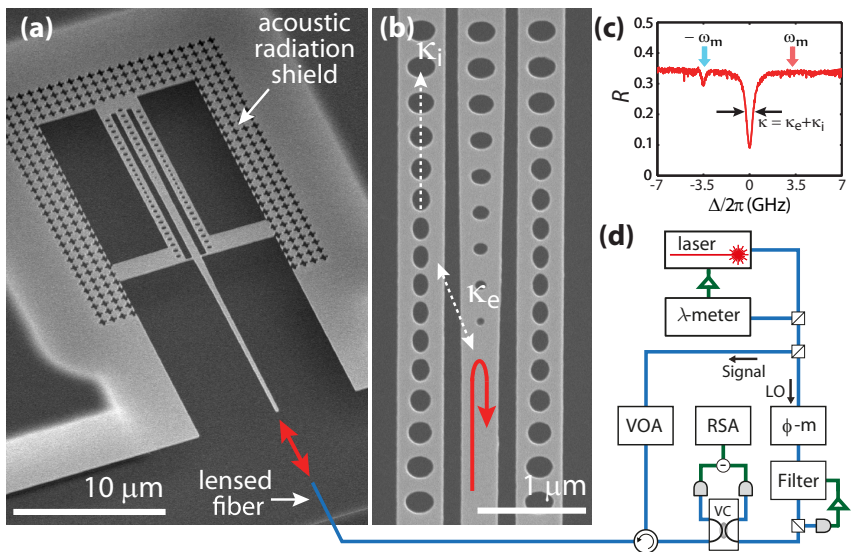

FIG. 1. (Color online) (a) SEM image of the OMC device. (b) Zoomed in SEM image showing details of the waveguide-cavity coupling region. (c) Normalized optical cavity reflection spectrum $(R)$. Detunings from resonance of $\Delta= \pm \omega_{\mathrm{m}} / 2 \pi= \pm 3.6 \mathrm{GHz}$ are denoted by the red and blue arrows, respectively. (d) Schematic of the fiber-based heterodyne receiver used to perform optical and mechanical spectroscopy of the OMC cavity. $\lambda$ meter: wave meter, $\varphi$-m: electro-optic phase modulator, VOA: variable optical attenuator, VC: variable coupler, RSA: real-time spectrum analyzer.

OMC cavity, collected by the lensed fiber, and detected on a photodetector is estimated to be $\eta_{\mathrm{cpl}}=34.7 \%$ [19].

The optical heterodyne detection scheme illustrated in Fig. 1(d) is used to measure the motion of the localized breathing mode at frequency $\omega_{\mathrm{m}} / 2 \pi=3.6 \mathrm{GHz}$. A high-power $(\sim$ $0.7 \mathrm{~mW}$ ) local oscillator (LO) sets the gain of the heterodyne receiver, and a low-power $(1 \mathrm{nW}-16 \mu \mathrm{W})$ optical signal beam is used to probe the OMC cavity. In order to selectively detect either the upper or lower motional sidebands generated on the optical signal beam, the LO frequency $\left(\omega_{\mathrm{LO}}\right)$ is shifted relative to that of the signal beam $\left(\omega_{\mathrm{s}}\right)$. For the measurements presented here, $\omega_{\mathrm{LO}}$ is adjusted such that the mechanical modulation beat frequency $\Omega=\omega_{\mathrm{LO}}-\left(\omega_{\mathrm{s}} \pm \omega_{\mathrm{m}}\right) \approx 2 \pi \times 50 \mathrm{MHz}$, placing a single motional sideband within the bandwidth $(100 \mathrm{MHz})$ of the photodetectors. In the case of a signal beam red-detuned from cavity resonance (frequency $\omega_{\mathrm{c}}$ ) by $\Delta \equiv \omega_{\mathrm{c}}-\omega_{\mathrm{s}}=$ $\omega_{\mathrm{m}}$, the resultant noise power spectral density (NPSD) as transduced on a spectrum analyzer yields a resonant mechanical signal component proportional to $S_{\mathrm{bb}}(\omega)=\langle n\rangle \gamma /[(\omega-$ $\left.\left.\omega_{\mathrm{m}}\right)^{2}+(\gamma / 2)^{2}\right][22]$. Here $\langle n\rangle$ is the phonon occupancy of the mechanical mode, and the total mechanical damping rate is given by $\gamma=\gamma_{\mathrm{i}}+\gamma_{\mathrm{OM}}$, where $\gamma_{\mathrm{i}}$ is the intrinsic damping rate of the mechanical resonator and $\gamma_{\mathrm{OM}}\left(\Delta=\omega_{\mathrm{m}}\right)=4 g_{0}^{2} n_{\mathrm{c}} / \kappa$ is the optomechanically induced damping rate produced by an intracavity photon number $n_{\mathrm{c}}$. For a blue-detuned probe $\left(\Delta=-\omega_{\mathrm{m}}\right)$, the resonant component of the NPSD is proportional to $\langle n\rangle+1$ and $\gamma=\gamma_{\mathrm{i}}-\gamma_{\mathrm{OM}}\left(\Delta=\omega_{\mathrm{m}}\right)$.

Mechanical spectroscopy is first performed at a fridge temperature of $T_{\mathrm{f}}=4 \mathrm{~K}$ in order to calibrate the optomechanical transduction. The coupling rate $g_{0}$ is determined by observing the dependence of the mechanical linewidth on $n_{\mathrm{c}}$ for both red $\left(\Delta=\omega_{\mathrm{m}}\right)$ and blue $\left(\Delta=-\omega_{\mathrm{m}}\right)$ laser-cavity detunings, as shown in Fig. 2(a). Above a threshold value, $n_{\mathrm{c}}>n_{\mathrm{thr}} \approx 1.5$, optical amplification and self-oscillation of
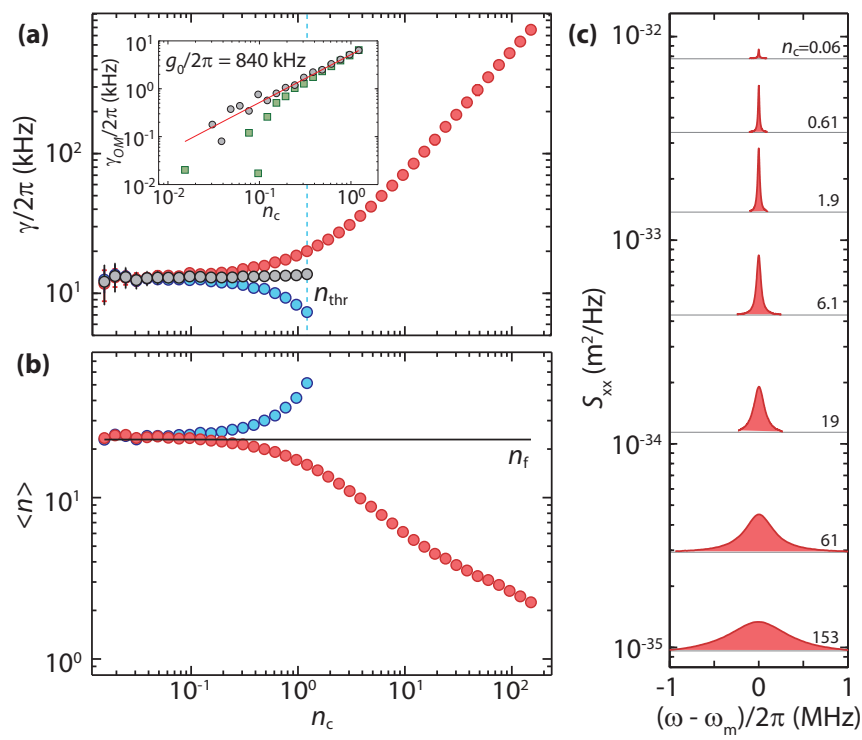

FIG. 2. (Color online) (a) Measured mechanical linewidth $\gamma$ for $\Delta=\omega_{\mathrm{m}}$ (red) and $-\omega_{\mathrm{m}}$ (blue) at a fridge temperature of $T_{\mathrm{f}}=4 \mathrm{~K}$. The vertical blue dashed line indicates the threshold $n_{\mathrm{c}}$ beyond which the mechanical resonance self-oscillates for $\Delta=-\omega_{\mathrm{m}}$, resulting in a $40 \mathrm{~dB}$ increase in the mechanical signal level. Black circles indicate the values of $\gamma_{i}$ obtained by taking the average of the detuned data. The inset shows $\gamma_{\mathrm{OM}}$ determined by subtracting $\gamma_{\mathrm{i}}$ from the red-detuned $\gamma$ (circles), and from cooperativity $C$ using the calibrated $\langle n\rangle$ (squares). A linear fit (red line) yields $g_{0} / 2 \pi=840 \mathrm{kHz}$. (b) Calibrated mechanical mode occupancy $\langle n\rangle$ versus intracavity photon number $n_{\mathrm{c}}$. Blue and red circles are measured with probe laser detunings $\Delta= \pm \omega_{\mathrm{m}}$, respectively. The mode occupancy $n_{\mathrm{f}}$ corresponding to $T_{\mathrm{f}}=4 \mathrm{~K}$ is indicated by a black solid line. (c) Series of red-detuned NPSD for range of $n_{\mathrm{c}}$. Here the NPSD is plotted as $S_{\mathrm{xx}}=x_{\mathrm{zp}}^{2} S_{\mathrm{bb}}$, where $x_{\mathrm{zpf}}=4.1 \mathrm{fm}$ is the zero-point amplitude of the breathing mode.

the mechanical resonator occurs for blue detuning. Below this value, the optomechanical damping $\gamma_{\mathrm{OM}}$ can be found from the difference between the red- and blue-detuned linewidths. A linear fit of the derived $\gamma_{\mathrm{OM}}$ versus $n_{\mathrm{c}}$ yields a coupling rate of $g_{0} / 2 \pi=840 \mathrm{kHz}$. Using this value of $g_{0}$ along with the optical detection efficiency, the mechanical mode occupancy versus $n_{\mathrm{c}}$ is calibrated from the area under the resonant part of the measured NPSD [see Figs. 2(b) and 2(c)]. At high $n_{\mathrm{c}}$ the mechanical mode is seen to be cooled, whereas at low $n_{\mathrm{c}}$ the calibrated occupancy saturates to a constant value in good agreement with the expected $4 \mathrm{~K}$ fridge occupancy (solid black line).

As the fridge temperature is lowered into the subkelvin range, a very different dependence of measured linewidth and mode occupancy on optical probe power is observed. In particular, the measured mechanical linewidth versus $n_{\mathrm{c}}$, shown in Fig. 3(a) for $T_{\mathrm{f}}=185 \mathrm{mK}$, increases with decreasing probe power below an apparent minimum at $n_{\mathrm{c}} \sim 1$. The measured linewidth at low power is also too large to explain the observed threshold of self-oscillation, $n_{\mathrm{thr}} \approx 0.1$. These inconsistencies indicate that the linewidth associated with the true energy decay rate of the mechanics is likely obscured in the time-averaged spectrum of Fig. 3(a) due to frequency 

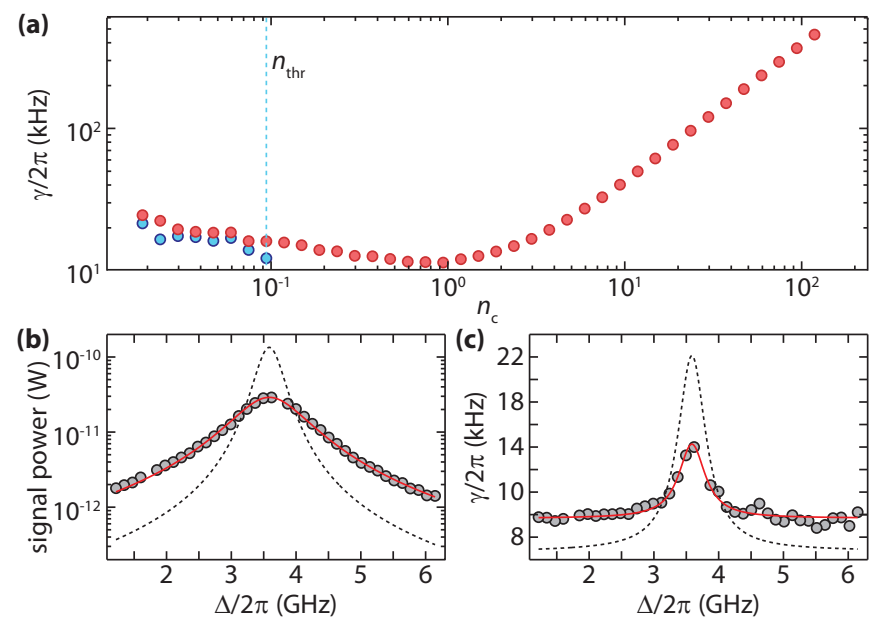

FIG. 3. (Color online) (a) Mechanical linewidth versus $n_{\mathrm{c}}$ for red- (red circles, $\Delta=\omega_{\mathrm{m}}$ ) and blue-detuned (blue circles, $\Delta=-\omega_{\mathrm{m}}$ ) probes at $T_{\mathrm{f}}=185 \mathrm{mK}$. The blue dashed line indicates the selfoscillation threshold. (b) Measured resonant signal power (circles) versus optical detuning, $\Delta$, for $n_{\mathrm{c}} \sim 1.5$. The red curve shows a best fit to the data, yielding $C=\gamma_{\mathrm{OM}} / \gamma_{\mathrm{i}}=3.9$ at $\Delta=\omega_{\mathrm{m}}$. Here we have assumed, to good approximation when $\omega_{\mathrm{m}} / \kappa \gg 1$ and $\left(\Delta-\omega_{\mathrm{m}}\right)^{2} \gg$ $\left(\Delta+\omega_{\mathrm{m}}\right)^{2}$, a back-action damping $\gamma_{\mathrm{OM}}(\Delta)=\left(4 g_{0}^{2} n_{\mathrm{c}} / \kappa\right)[1+4(\Delta-$ $\left.\left.\omega_{\mathrm{m}}\right)^{2} / \kappa^{2}\right]^{-1}$, cooled mode occupancy $\langle n\rangle \propto\left[1+\gamma_{\mathrm{OM}}(\Delta) / \gamma_{\mathrm{i}}\right]^{-1}$, and heterodyne resonant signal power $\propto\langle n\rangle \gamma_{\mathrm{OM}}$. The black dashed curve shows the expected signal in the absence of back-action cooling $(C \rightarrow 0)$, consistent with assuming $\gamma_{\mathrm{i}}$ is equal to the measured timeaveraged linewidth. (c) Corresponding measured (circles) mechanical linewidth versus $\Delta$. The red curve is a fit with $C\left(\Delta=\omega_{\mathrm{m}}\right)$ constrained $(=3.9)$, but assuming a Voigt line shape with additional Gaussian frequency jitter term. The dashed black curve is the best fit for $C$ constrained (=3.9) but with no additional frequency jitter term.

jitter $[23,24]$. Due to the long averaging times (minutes to hours) required at low optical probe power, direct observation of the mechanical frequency jitter is not possible. Indirect confirmation of the frequency jitter is ascertained by studying the detuning $(\Delta)$ dependence of the transduced signal power and linewidth for a fixed $n_{\mathrm{c}} \sim 1.5$, as shown in Fig. 3(b). Such a measurement keeps constant any effects such as optical heating or frequency jitter that might depend on the intracavity photon number. The resulting fit to the data (red curves; see caption for details), yields $\gamma_{\mathrm{i}} / 2 \pi=2.3 \mathrm{kHz}$ for the intrinsic mechanical energy decay rate, $\gamma_{\mathrm{G}} / 2 \pi=6.1 \mathrm{kHz}$ for the Gaussian frequency jitter, and $g_{0} / 2 \pi=805 \mathrm{kHz}$, consistent with the $T_{\mathrm{f}}=4 \mathrm{~K}$ value. In what follows we use additional on-resonance heating measurements to determine $\gamma_{i}$ over the entire range of $n_{\mathrm{c}}$.

Heating of the mechanical mode by optical absorption becomes significant at subkelvin temperatures due to the sharp drop in thermal conductance with temperature [25]. The source of optical absorption in our structures is most likely due to electronic defect states at the surface of Si [26,27]. This heating mechanism is investigated at $T_{\mathrm{f}}=10 \mathrm{mK}$, for which $n_{\mathrm{f}}$ is negligible, by measuring $\langle n\rangle$ and $\gamma$ using an optically resonant probe $\left(\Delta=0 ; \gamma_{\mathrm{OM}}=0\right)$. We observe in Fig. 4(a) that $\langle n\rangle \propto n_{\mathrm{c}}^{1 / 4}$. This weak power-law dependence is consistent with indirect coupling of the breathing mode to an optically generated bath with thermal conductance scaling as $G_{\text {th }} \sim T^{3}$. Phonons with wavelengths small relative to the dimensions of the cavity structure are expected to have such a conductance scaling [28], although their estimated escape time from the nanobeam $\left(\gamma_{\mathrm{THz}}^{-1} \sim 1-10 \mathrm{~ns}\right)$ suggests that they must quickly come into thermal equilibrium. Given the slow rates of most bulk relaxation processes [29], this fast thermalization is likely due to a relatively large degree of diffusive and inelastic scattering at the surfaces of the patterned nanobeam [30].

Based on these on-resonance observations, a proposed microscopic model for the optical absorption heating and damping is illustrated in Fig. 4(c). Here the long-lived breathing mode is weakly coupled $\left(\gamma_{0}\right)$ through the phononic shield to the exterior fridge environment, and is locally coupled via phonon-phonon scattering $\left(\gamma_{\mathrm{p}}\right)$ [31] to the optically generated high frequency phonons within the acoustic cavity. A phenomenological model based upon this microscopic picture is shown schematically in Fig. 4(d). We parametrize the coupling of the mechanical resonator to the separate thermal baths by decomposing the mechanical damping rate into $\gamma_{\mathrm{L}}=$ $\gamma_{0}+\gamma_{\mathrm{p}}+\gamma_{\mathrm{OM}}$, where the fridge bath (occupancy $n_{\mathrm{f}}$ ) couples at rate $\gamma_{0}$, the optical-absorption-induced bath (temperature $T_{\mathrm{p}}$, occupancy $n_{\mathrm{p}}$ at $\omega_{\mathrm{m}}$ ) couples at rate $\gamma_{\mathrm{p}}$, and the intracavity laser field (effective zero-temperature bath) couples at rate $\gamma_{\mathrm{OM}}$. The resulting average mechanical mode occupation is then given by $\langle n\rangle\left(n_{\mathrm{c}}\right)=\left[\gamma_{0} n_{\mathrm{f}}+\gamma_{\mathrm{p}}\left(T_{\mathrm{p}}\right) n_{\mathrm{p}}\left(T_{\mathrm{p}}\right)\right] /\left[\gamma_{0}+\gamma_{\mathrm{p}}\left(T_{\mathrm{p}}\right)+\gamma_{\mathrm{OM}}\left(n_{\mathrm{c}}\right)\right]$, where $T_{\mathrm{p}}\left(n_{\mathrm{c}}\right)$.

The calibrated mechanical mode occupation for a reddetuned $\left(\Delta=\omega_{\mathrm{m}}\right)$ probe is plotted against $n_{\mathrm{c}}$ in Fig. 4(e) for $T_{\mathrm{f}}=10 \mathrm{mK}$ (purple) and $635 \mathrm{mK}$ (green). Both curves exhibit a series of heating and cooling trends, and in fact coincide for $n_{\mathrm{c}} \gtrsim 1$. At the lowest optical probe powers $\left(n_{\mathrm{c}}=0.016\right)$ and lowest fridge temperature $\left(T_{\mathrm{f}}=10 \mathrm{mK}\right)$, the calibrated phonon occupancy reaches a minimum $\langle n\rangle=0.98 \pm 0.11$, corresponding to $T \approx 270 \mathrm{mK}$. The complex behavior of these two cooling curves can be understood by comparing to the proposed phenomenological model. For this model $n_{\mathrm{f}}$ is taken to correspond to the measured $T_{\mathrm{f}}, n_{\mathrm{p}}\left(n_{\mathrm{c}}\right)$ is ascertained by extrapolating the on-resonance measurement of $\langle n\rangle$ [Fig. 4(a)], and $\gamma_{\mathrm{OM}}\left(n_{\mathrm{c}}\right)$ is found from the fit value $g_{0} / 2 \pi=840 \mathrm{kHz}$ to the high power region of the red-detuned mechanical linewidth [red circles in Fig. 4(b)]. Assuming a common $\gamma_{\mathrm{p}}\left(n_{\mathrm{c}}\right)$ and $\gamma_{0}$, the resulting $\gamma_{\mathrm{i}}\left(n_{\mathrm{c}}\right)$ curve that best fits the measured $\langle n\rangle$ data for both $T_{\mathrm{f}}=10$ and $635 \mathrm{mK}$ fridge temperatures is plotted in Fig. 4(b) (blue circles). Also shown in Fig. 4(b) are the best-fit value of the coupling to the fridge bath $\gamma_{0} / 2 \pi=306 \pm$ $28 \mathrm{~Hz}$ (dashed black horizontal curve) and a smooth spline curve fit to the inferred values of $\gamma_{\mathrm{p}}\left(n_{\mathrm{c}}\right)$ (red solid curve). A plot of the best-fit model is shown alongside the measured $\langle n\rangle$ cooling curves in Fig. 4(e). In addition to the good agreement of the model for both fridge temperatures, the inferred intrinsic energy damping rate is also consistent with the measured self-oscillation threshold [Fig. 3(a)]. At the lowest probe powers $\left(n_{\mathrm{c}}=0.016\right)$, the energy damping mechanical $Q$ factor reaches an impressively high value of $Q_{\mathrm{m}}=9 \times 10^{6}$.

Alongside the calibrated mode occupancy $\langle n\rangle$, we have also measured the sideband asymmetry $\xi$ shown in the inset to Fig. 4(e). The sideband asymmetry is defined as $\xi=I_{-} / I_{+}-1$ [32], where $I_{ \pm}$is the area under the Lorentzian part of the NPSD for an optical probe with 
(a)

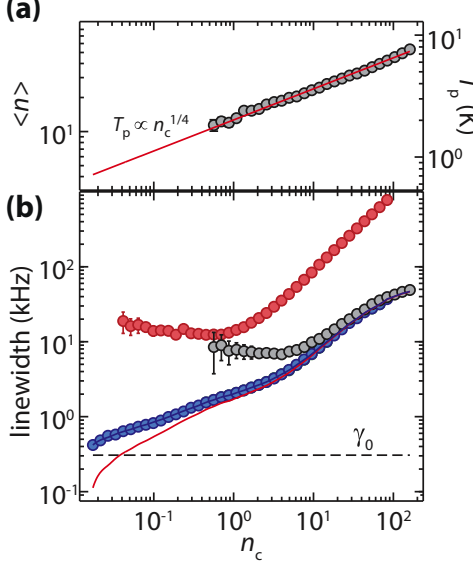

(c)

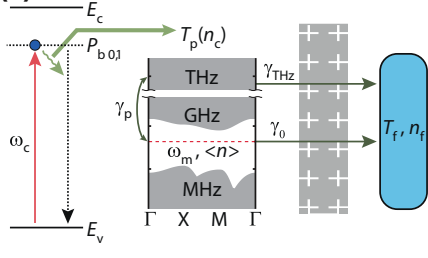

(d)

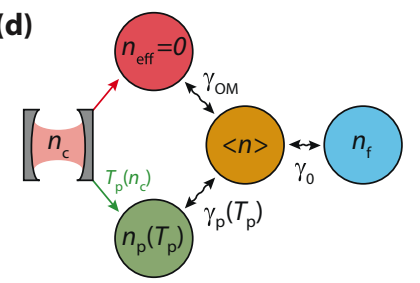

(e)

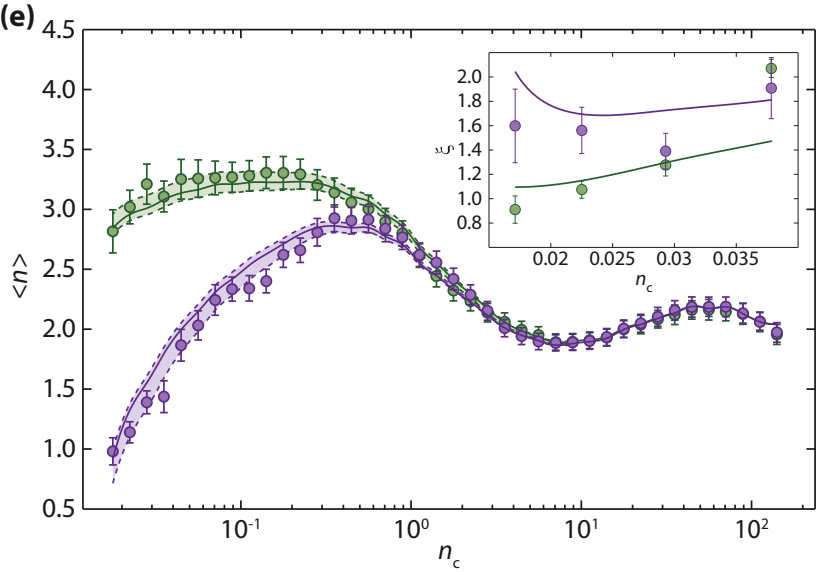

FIG. 4. (Color online) (a) Measured phonon occupancy versus intracavity photon number for a resonant probe at $T_{\mathrm{f}}=10 \mathrm{mK}$. The red line shows a power-law fit to the occupancy. The right axis shows the equivalent bath temperature, $T_{\mathrm{p}}$. (b) Measured mechanical linewidth versus $n_{\mathrm{c}}$ for resonant (gray circles) and red-detuned (red circles) probes. The blue circles show the best-fit values of $\gamma_{\mathrm{i}}=\gamma_{0}+\gamma_{\mathrm{p}}$ to both $T_{\mathrm{f}}=10$ and $635 \mathrm{mK}$ data sets. The best-fit value for $\gamma_{0}$ and a smooth curve fit to the inferred $\gamma_{\mathrm{p}}$ are shown as a black dashed line and a solid red line, respectively. (c) Diagram illustrating the proposed model of optical-absorption-driven heating of the mechanics and (d) schematic showing the various baths coupled to the localized mechanical mode (see text for details). (e) Calibrated mode occupancy versus $n_{\mathrm{c}}$ for $T_{\mathrm{f}}=10 \mathrm{mK}$ and $635 \mathrm{mK}$. Best fits to both temperature data sets using the proposed heating model are shown as solid curves, with shaded regions representing the variation in the fit for $\gamma_{0} / 2 \pi=306 \pm 28 \mathrm{~Hz}$. The inset shows the measured asymmetry $\xi$ as a function of $n_{\mathrm{c}}$, with the prediction of the best-fit model shown as solid curves. $T_{\mathrm{f}}=10 \mathrm{mK}$ data (fits) are shown as purple circles (solid curves). $T_{\mathrm{f}}=635 \mathrm{mK}$ data (fits) are shown as green circles (solid curves).

detuning $\Delta= \pm \omega_{\mathrm{m}}$. The asymmetry is sensitive to both the absolute mode occupancy and to the sum of $\gamma_{0}$ and $\gamma_{\mathrm{p}}$ through the cooperativity $C=\gamma_{\mathrm{OM}} /\left(\gamma_{0}+\gamma_{\mathrm{p}}\right)$. Good correspondence can also be seen between the best-fit model (solid curves) and the measured $\xi$ (circles).

Although significant work remains to determine the exact microscopic details of the optical absorption heating and frequency jitter observed in the measurements of the quasi1D OMC cavity studied here, there are nonetheless several interesting points to note. First, from the measured timeaveraged mechanical linewidth and optically induced bath temperature $\left(T_{\mathrm{p}}\right)$ in Fig. 4 we find that the frequency noise of the mechanical resonator drops with increasing temperature as $T_{\mathrm{p}}^{-0.9}$ [19]. Such a frequency noise behavior is similar to that found for two-level systems coupled to superconducting microwave resonators [24], and may be due to native oxide formation at the Si surfaces. Secondly, the phononic shield provides excellent mechanical isolation of the breathing mode, while at the same time providing good mechanical coupling to the fridge bath for heat carrying phonons above the acoustic band gap. Thirdly, although lower phonon occupancies could have been measured using thinner phononic shields, effectively increasing the coupling rate $\gamma_{0}$ to the fridge bath at $T_{\mathrm{f}}$, this would come with a commensurate reduction in cooperativity $C=\gamma_{\mathrm{OM}} / \gamma_{\mathrm{i}}$. Coherent quantum interactions between the optical cavity field and the mechanical resonator require $C>1$ and $\langle n\rangle<1$, and although the devices of this work closely approach this limit, a move to quasi-2D Si OMC devices [33] with orders of magnitude larger thermal conductance should enable future work in the quantum regime as envisioned in recent proposals [13,15-17].

The authors would like to thank Michael Roukes, Ron Lifshitz, and Michael Cross for helpful discussions regarding the proposed thermal model, as well as Jasper Chan, Witlef Wiezcorek, and Jason Hoelscher-Obermaier for support in the early stages of the experiment. This work was supported by the DARPA ORCHID and MESO programs, the Institute for Quantum Information and Matter, an NSF Physics Frontiers Center with support of the Gordon and Betty Moore Foundation, and the Kavli Nanoscience Institute at Caltech. A.S.N. acknowledges support from NSERC. S.G. was supported by a Marie Curie International Outgoing Fellowship within the 7th European Community Framework Programme. S.M.M., J.D.C., and S.G. contributed equally to this work.
[1] V. Braginsky and A. Manukin, Measurement of Weak Forces in Physics Experiments (University of Chicago Press, Chicago, 1977).

[2] The LIGO Scientific Collaboration, Nat. Phys. 7, 962 (2011).

[3] A. G. Krause, M. Winger, T. D. Blasius, Q. Lin, and O. Painter, Nat. Photon. 6, 768 (2012).

[4] T. Rocheleau, T. Ndukum, C. Macklin, J. B. Hertzberg, A. A. Clerk, and K. C. Schwab, Nature (London) 463, 72 (2010).
[5] J. D. Teufel, T. Donner, D. Li, J. W. Harlow, M. S. Allman, K. Cicak, A. J. Sirois, J. D. Whittaker, K. W. Lehnert, and R. W. Simmonds, Nature (London) 475, 359 (2011).

[6] J. Chan, T. P. M. Alegre, A. H. Safavi-Naeini, J. T. Hill, A. Krause, S. Gröblacher, M. Aspelmeyer, and O. Painter, Nature (London) 478, 89 (2011).

[7] E. Verhagen, S. Deléglise, S. Weis, A. Schliesser, and T. J. Kippenberg, Nature (London) 482, 63 (2012). 
[8] M. Eichenfield, J. Chan, R. M. Camacho, K. J. Vahala, and O. Painter, Nature (London) 462, 78 (2009).

[9] S. Gröblacher, J. B. Hertzberg, M. R. Vanner, G. D. Cole, S. Gigan, K. C. Schwab, and M. Aspelmeyer, Nat. Phys. 5, 485 (2009).

[10] J. D. Thompson, B. M. Zwickl, A. M. Jayich, F. Marquardt, S. M. Girvin, and J. G. E. Harris, Nature (London) 452, 72 (2008).

[11] A. H. Safavi-Naeini and O. Painter, Opt. Express 18, 14926 (2010).

[12] D. Chang, A. H. Safavi-Naeini, M. Hafezi, and O. Painter, New J. Phys. 13, 023003 (2011).

[13] M. Schmidt, M. Ludwig, and F. Marquardt, New J. Phys. 14, 125005 (2012).

[14] S. J. M. Habraken, K. Stannigel, M. D. Lukin, P. Zoller, and P. Rabl, New J. Phys. 14, 115004 (2012).

[15] A. Tomadin, S. Diehl, M. D. Lukin, P. Rabl, and P. Zoller, Phys. Rev. A 86, 033821 (2012).

[16] M. Ludwig and F. Marquardt, Phys. Rev. Lett. 111, 073603 (2013).

[17] M. Schmidt, V. Peano, and F. Marquardt, arXiv:1311.7095.

[18] J. Chan, Ph.D. thesis, California Institute of Technology, 2012.

[19] See Supplemental Material at http://link.aps.org/supplemental/ 10.1103/PhysRevA.90.011803 for details of device design, fabrication, and characterization.
[20] V. R. Almeida, R. R. Panepucci, and M. Lipson, Opt. Lett. 28, 1302 (2003).

[21] J. D. Cohen, S. M. Meenehan, and O. Painter, Opt. Express 21, 11227 (2013).

[22] A. H. Safavi-Naeini, J. Chan, J. T. Hill, S. Gröblacher, H. Miao, Y. Chen, M. Aspelmeyer, and O. Painter, New J. Phys. 15, 035007 (2013).

[23] Y. T. Yang, C. Callegari, X. L. Feng, and M. L. Roukes, Nano Lett. 11, 1753 (2011).

[24] J. Gao, Ph.D. thesis, California Institute of Technology, 2008.

[25] M. G. Holland, Phys. Rev. 132, 2461 (1963).

[26] A. Stesmans, Appl. Phys. Lett. 68, 2076 (1996).

[27] M. Borselli, T. J. Johnson, C. P. Michael, M. D. Henry, and O. Painter, Appl. Phys. Lett. 91, 131117 (2007).

[28] R. Chen, A. I. Hochbaum, P. Murphy, J. Moore, P. Yang, and A. Majumdar, Phys. Rev. Lett. 101, 105501 (2008).

[29] N. Mingo, Phys. Rev. B 68, 113308 (2003).

[30] J. B. Hertzberg, M. Aksit, O. O. Otelaja, D. A. Stewart, and R. D. Robinson, Nano Lett. 14, 403 (2014).

[31] P. S. Zyryanov and G. G. Taluts, J. Exp. Theor. Phys. 22, 1326 (1966).

[32] A. H. Safavi-Naeini, J. Chan, J. T. Hill, Thiago P. Mayer Alegre, A. Krause, and O. Painter, Phys. Rev. Lett. 108, 033602 (2012).

[33] A. H. Safavi-Naeini, J. T. Hill, S. Meenehan, J. Chan, S. Gröblacher, and O. Painter, Phys. Rev. Lett. 112, 153603 (2014). 